Fernando Antonio Mourão Valejo

Daniel Guimarães TIEZZI ${ }^{2}$

Gisele Alborguetti Nal

Relato de caso

Palavras-chave

Fibromatose abdominal Fibromatose agressiva/cirurgia Fibromatose agressiva/diagnóstico

Fibromatose agressiva/etiologia

Neoplasias abdominais

Fibroblastos

Relatos de casos

Keywords

Abdominal neoplasms Fibromatosis, aggressive/surgery Fibromatosis, aggressive/diagnosis Fibromatosis, aggressive/etiology Fibromatosis, abdominal Fibroblasts Case reports

\section{Tumor desmoide abdômino-pélvico}

\author{
Abdominopelvic desmoid tumor
}

\section{Resumo}

tumor desmoide é uma neoplasia não capsulada, localmente agressiva, originária dos fibroblastos dos tecidos músculo-aponeuróticos. Embora ele não tenha comportamento maligno, tal como capacidade de gerar metástases ou de invasão, o tumor desmoide tem uma alta capacidade de crescimento local, causando deformidades nos órgãos adjacentes, dor e, eventualmente, disfunção orgânica, dependendo da área envolvida. Relatamos o caso de um tumor desmoide intra-abdominal de grandes proporções, invadindo órgãos pélvicos em uma paciente de 53 anos. A neoplasia foi totalmente extirpada e, atualmente, cinco anos após a cirurgia, a paciente encontra-se sem sinal clínico ou radiológico de recidiva da lesão.

\section{Abstract}

Desmoid tumor is a noncapsulated neoplasia, locally aggressive, originated from the fibroblasts of the musculo-aponeurotic tissues. Even though with no malignant behavior, such as the ability of generating metastasis or of invasion, the desmoid tumor has a high tendency for local growth, causing deformities in the adjacent organs, pain and sometimes organ dysfunction, depending on the area involved. We report the case of a large intra-abdominal desmoid tumor, invading pelvic organs in a 53 year-old patient. The neoplasia has been totally extirpated and, at the moment, five years after the surgery, the patient presents no clinical or radiologic sign of lesion relapse.
Correspondência:

Fernando Antonio Mourão Valejo Rua Fernão Dias, 940, apto. 501 CEP 19023-280 - Presidente Prudente (SP), Brasil E-mail: fvalejo@ig.com.br

Recebido 22/10/08

Universidade do Oeste Paulista - UNOESTE - Presidente Prudente (SP), Brasil.

'Professor do Departamento de Ginecologia e Obstetrícia da Universidade do Oeste Paulista - UNOESTE - Presidente Prudente (SP), Brasil.

2 Professor do Departamento de Ginecologia e Obstetrícia da Faculdade de Medicina de Ribeirão Preto da Universidade de São Paulo - FMRP-USP - Ribeirão Preto (SP), Brasil.

3 Professora do Departamento de Patologia da Universidade do Oeste Paulista - UNOESTE - Presidente Prudente (SP), Brasil. 


\section{Introdução}

O tumor desmoide, também conhecido como fibromatose profunda, é um tumor não capsulado, localmente agressivo, originário dos fibroblastos dos tecidos músculoaponeuróticos. Foi descrito pela primeira vez em 1832, recebendo, posteriormente o nome de desmoide para indicar sua aparência macroscópica (do grego "desmos", significa tendão ou ligamento $)^{1}$. Embora não tenha comportamento maligno, tal como capacidade de gerar metástases ou de invasão, o tumor desmoide tem uma alta capacidade de crescimento local, causando deformidades nos órgãos adjacentes, dor e, eventualmente, disfunção orgânica, dependendo da área envolvida ${ }^{2-4}$. Além disso, mesmo sendo tratado adequadamente com excisão cirúrgica, respeitando-se margens de segurança, apresenta alta taxa de recorrência local que pode variar de 25 a $77 \%$ ao ano ${ }^{5,6}$. Pode surgir praticamente em qualquer idade e aparecer em quase todos os segmentos corporais ${ }^{7}$. Embora os achados histológicos sejam bem característicos, atualmente, todo conhecimento a seu respeito se baseia apenas em alguns relatos isolados e em algumas pequenas séries de casos.

\section{Relato do caso}

Paciente com 53 anos, branca, empregada doméstica, natural de Aracaju, Sergipe, procedente de Mirante do Paranapanema, São Paulo, foi referenciada ao ambulatório de ginecologia do Hospital Universitário de Presidente Prudente no dia 20 de dezembro de 2002. Relatava uma massa de grande volume na fossa ilíaca esquerda. Relatava dor, associada à massa, que irradiava para o hipogástrio, intermitente, latejante, intensa, sem fatores de piora e com melhora ao uso de anti-inflamatórios não-hormonais. Relatava antecedentes

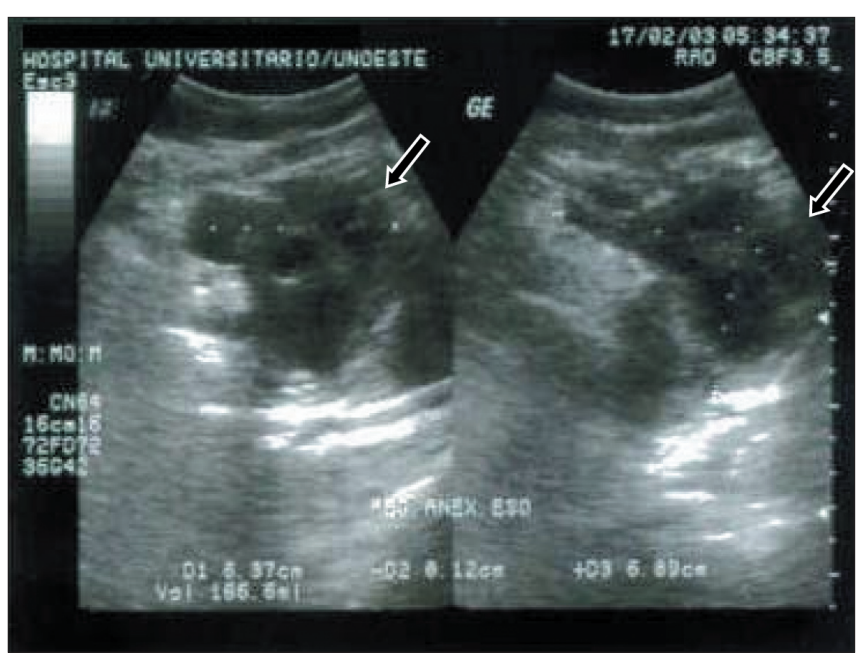

Figura 1 - Imagem ultrassonográfica da região anexial esquerda. Não há caracterização clássica dos órgãos anexiais. Nota-se imagem de ecogenicidade mista, forma indefinida, contornos irregulares, evidenciando o aspecto infiltrativo e amorfo da lesão (setas). de hipertensão arterial, com uso de captopril 50mg/dia, e tabagismo de dez cigarros por dia. Negava diabetes ou antecedentes oncológicos. Teve seis partos normais e uma cesariana anterior (G8, P7, A1), sendo o último parto há 13 anos (cesariana). A última menstruação ocorrera havia três meses. Não fazia uso de contraceptivo ou outra medicação hormonal. Relatava ter parceiro único.

Ao exame físico, notava-se massa de consistência fibroelástica, regular e globosa na fossa ilíaca esquerda, fixa aos planos adjacentes e dolorosa à palpação. O exame ginecológico não revelava alterações significativas.

A ultrassonografia endovaginal mostrou útero de $80,7 \mathrm{~cm}^{3}$, endométrio normoecoide com $8 \mathrm{~mm}$ de espessura, ovário direito sem alterações e ovário esquerdo apresentando-se aumentado às custas de um tumor císticosólido de 4,2 cm de diâmetro no seu maior eixo. A Figura 1 mostra o aspecto ultrassonográfico da lesão. A dosagem de CA 125 foi $16,8 \mu / \mathrm{mL}$ (negativa).

A paciente foi encaminhada à laparotomia, onde se encontrou tumor de consistência endurecida, superfície mal delimitada de aproximadamente $15 \mathrm{~cm}$ de diâmetro em topografia anexial esquerda, infiltrando grande parte da bexiga urinária, comprometendo inclusive a mucosa do órgão, músculo reto abdominal e cólon sigmoide. $\mathrm{O}$ exame intraoperatório revelou uma neoplasia mesenquimal de etiologia a esclarecer. Foi sugerido aguardar o exame histológico definitivo.

Foi realizada excisão completa da lesão com histerectomia total, anexectomia bilateral, ressecção parcial do músculo reto abdominal, cistectomia parcial e ressecção parcial do cólon sigmoide, com anastomose términoterminal primária. A paciente permaneceu internada por 19 dias, recebendo alta em boas condições.

O estudo anatomopatológico dos espécimes ressecados revelou fibromatose abdominal e pélvica, comprometendo e distorcendo os anexos esquerdos, parede abdominal, parede do cólon sigmoide e parede vesical. As Figuras 2 e 3 representam o aspecto histológico do tumor.

Após retorno pós-operatório, a paciente foi acompanhada semestralmente durante um período de cinco anos, sem sinal clínico-radiológico de recidiva da lesão e com tomografia abdominal normal. Em dezembro de 2007, a paciente encontrava-se ictérica. Foi submetida a uma endoscopia digestiva alta com biópsia de papila duodenal e diagnóstico de adenocarcinoma de papila. Foi, então, tratada em outro serviço. No último atendimento, em 11 de dezembro de 2008, a paciente encontrava-se assintomática, sem sinal clínico de recidiva das lesões.

A elaboração deste relato de caso foi aprovada pela Comissão de Ética do Hospital Universitário da Universidade do Oeste Paulista (UNOESTE). A paciente assinou termo de consentimento livre e esclarecido, autorizando sua publicação. 


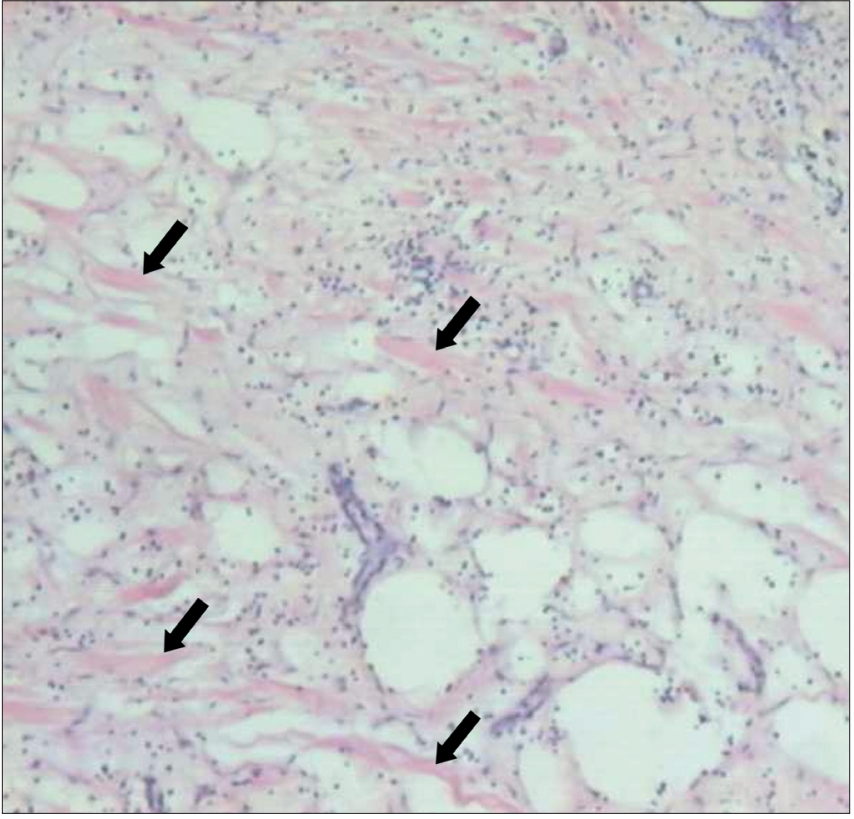

Figura 2 - Depósito de fibras colágenas (setas) com vários capilares entremeados por infiltrado inflamatório mononuclear, por vezes formando folículos linfoides (hematoxilinaeosina, 100X).

\section{Discussão}

O tumor desmoide corresponde a $0,03 \%$ de todas as neoplasias e menos de $3 \%$ de todos os tumores de tecidos moles. A incidência estimada na população geral é de dois a quatro casos por milhão de pessoas por ano ${ }^{8}$. Ele se desenvolve como resultado de uma proliferação progressiva de fibroblastos. Em alguns pacientes ele progride rápida e agressivamente, porém em outros ele é mais indolente e pode permanecer estável sem causar qualquer morbidade no futuro? ${ }^{9}$. No entanto, a maioria dos tumores desmoides são neoplasias de crescimento lento, não metastatizantes, localmente agressivas, que invadem tecidos e órgãos ou comprimem estruturas ${ }^{10} . \mathrm{Na}$ mulher, a regressão espontânea da doença está associada à menopausa ou à ooforectomia, o que sugere uma relação entre tumor desmoide e estrogênio ${ }^{10,11}$.

Infelizmente, algumas lesões progridem para múltiplas recorrências seguidas de várias ressecções cirúrgicas. Cerca de $10 \%$ dos tumores desmoides resolvem espontaneamente; $30 \%$ permanecem em ciclos de progressão e resolução; $50 \%$ permanecem estáveis após o diagnóstico e $10 \%$ progridem rapidamente ${ }^{12}$. Sendo assim, apesar da aparência histológica benigna do tumor desmoide, seu comportamento biológico é característico de lesões malignas, podendo inclusive levar a paciente à morte9. Sua alta capacidade de recorrência e de invasão de órgãos e vasos vizinhos pode levar a uma obstrução de órgão ou estrutura vital, o que dificulta seu manejo. As complicações podem ir desde alterações como hidronefrose, obstruções intestinais e formações fistulares, até hemorragias

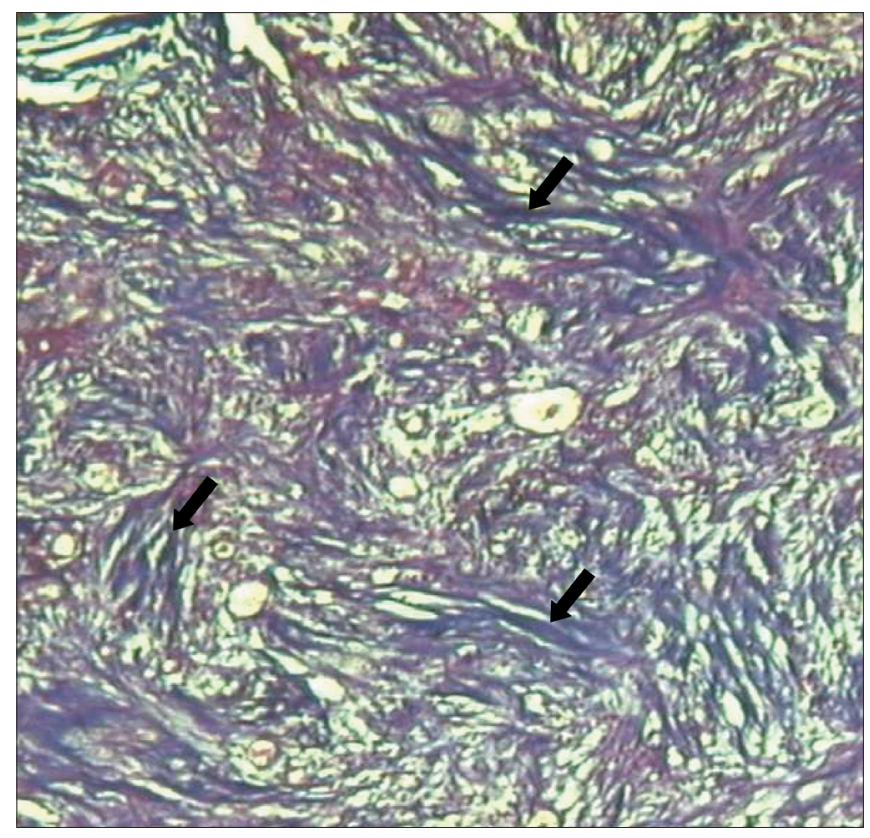

Figura 3 - Coloração tricrômio de Masson. A coloração azulada mostra a grande proeminência de tecido fibroso no tumor (setas).

internas, infecções, perfurações intestinais, peritonites, entre outros.

Dependendo da sua localização, os tumores desmoides podem ser classificados em extra-abdominais e abdominais. Os extra-abdominais são mais frequentemente observados em áreas como ombro, parede torácica e joelho, e estão sempre confinados à musculatura ou aponeurose. $\mathrm{O}$ tipo abdominal é mais comum e é dividido em superficial (ou de parede), quando o tumor se desenvolve junto à musculatura abdominal, e em intra-abdominal, quando o tumor se desenvolve dentro da cavidade abdominal ou pélvica. Pode acontecer de múltiplos tumores desenvolverem-se em um mesmo paciente (doença multicêntrica), mas tendem a aparecer em uma mesma região ${ }^{8}$.

O tumor desmoide pode ser, ainda, esporádico (idiopático) ou familiar. A forma esporádica é frequentemente de localização extra-abdominal e normalmente passível de ressecção. A forma familiar da doença está normalmente associada à polipose familiar, observada em 10 a 15\% dos pacientes, e está relacionada a alterações genéticas específicas $^{13}$. A forma esporádica é mais comum nas mulheres do que nos homens, em proporções que variam de 2:1 até 5:1. Na infância, a incidência é igual em ambos os sexos. Não há diferença entre os sexos na incidência da forma hereditária do tumor. É frequentemente observado em pacientes jovens (10 a 40 anos). Na infância, representa menos de $1 \%$ de todos os tumores infantis e tende a ocorrer principalmente em crianças com história familiar de polipose intestinal ou câncer de cólon hereditário ${ }^{10}$. Em um recente estudo, a média de idade dos pacientes $(n=60)$ com tumor desmoide foi de 41,3 anos ${ }^{14}$. 
O miofibroblasto é a célula considerada responsável pela origem do tumor desmoide. Os mecanismos do desenvolvimento e regulação do seu crescimento não são conhecidos. Há evidências de que hormônios, especialmente os estrogênios, possam, de alguma forma, participar desses mecanismos ${ }^{11}$. Essas evidências são confirmadas pela maior incidência de tumor desmoide em mulheres durante sua vida reprodutiva, pela aparente tendência dos tumores a se desenvolverem durante a gestação ou logo em seguida, pelo seu ocasional desaparecimento após a menopausa, pela produção laboratorial de lesões similares em animais submetidos à administração de estrogênio e pelo benefício da terapia antiestrogênica em pacientes portadoras desse tipo de tumor ${ }^{15}$.

Os sinais clínicos e sintomas dependem da localização do tumor, da extensão, do tamanho da massa e da velocidade de crescimento da lesão ${ }^{15}$. O tumor desmoide apresenta consistência endurecida à palpação. $\mathrm{O}$ corte transmite uma sensação áspera e arenosa e o aspecto interno revela uma superfície clara, brilhante, grosseiramente trabeculada, semelhante a tecido cicatricial ${ }^{10}$. Alguns tumores intra-abdominais podem invadir diretamente a parede abdominal, sendo chamados de tumores "transabdominais", dificilmente ressecáveis ${ }^{13}$.

Macroscopicamente, os tumores desmoides são levemente circunscritos, infiltram o tecido vizinho e não apresentam cápsula verdadeira. Microscopicamente, são caracterizados por proliferação de células fusiformes alongadas, de aparência uniforme em meio a estroma colágeno, muitas vezes semelhante à queloide, com vascularização variável. As células normalmente são pequenas, com núcleos bem definidos e citoplasma claro não apresentando mitoses ou atipias. Possuem núcleos pálidos com cromatina uniforme e nucléolos inconspícuos ${ }^{16}$. Há pouco contato entre as células devido à abundante quantidade de colágeno em torno das células neoplásicas. Macrófagos, células gigantes e linfócitos estão presentes na periferia. Células musculares podem ser encontradas junto ao tumor. À imunoistoquímica, encontra-se positividade para marcadores de células musculares como vimentina, actina de músculo liso e desmina ${ }^{17}$. O termo "fibromatose agressiva" determina a intenção de descrever a celularidade marcante e a agressividade local da lesão. Frequentemente, esses tumores infiltram tecidos vizinhos, porém não metastatizam ${ }^{15}$. Na microscopia eletrônica, as células em fusos se parecem com miofibroblastos. Por ser uma lesão infiltrativa, o principal diagnóstico diferencial é com fibrossarcoma. Porém, a ausência de atipias celulares e mitoses frequentes, associada à positividade para marcadores de células musculares, excluem esse diagnóstico ${ }^{16}$. Alguns autores têm defendido a possibilidade da realização do diagnóstico através da punção citológica, caracterizando as células do tumor através de dados microscópicos e imunoistoquímicos ${ }^{7}$. Porém, os mesmos autores reconhecem a preferência pelo diagnóstico histológico através da biópsia incisional ou excisional.

Lesões da parede abdominal normalmente formam massas palpáveis ou visíveis, embaixo da pele, indolores, fixas e lisas, infiltrando a musculatura adjacente, frequentemente causando sua degeneração ${ }^{10}$. Dor ou parestesias podem aparecer como resultado da compressão de nervos ou musculatura adjacente. Eventualmente, são achados incidentais durante uma cirurgia ou algum exame de imagem $^{10}$.

Métodos de imagem podem auxiliar o diagnóstico do tumor desmoide, delimitando a extensão da lesão e sua relação com os órgãos adjacentes e outras estruturas anatômicas. Essa informação é particularmente importante na determinação da tática cirúrgica.

Definir o tratamento ideal para pacientes com tumor desmoide é uma tarefa árdua, já que, por se tratar de uma patologia rara, existem poucas publicações consistentes na literatura mundial, sendo que, em sua maioria, apresenta pequeno número de casos, dificultando o estabelecimento de conclusões. Os estudos encontrados são todos retrospectivos e, a princípio, devem ser vistos de forma cautelosa.

A principal dificuldade no tratamento do tumor desmoide é devida ao fato de que esses tumores são histologicamente benignos, mas possuem uma alta taxa de recorrência local ${ }^{10}$. Os efeitos do tratamento são frequentemente confundidos pela história natural da doença, onde alguns tumores aparentemente regridem ou permanecem estáveis mesmo sem tratamento ${ }^{12}$.

Observar tem sido a conduta de escolha para pacientes assintomáticos com tumores desmoides pequenos que não invadem ou comprimem estruturas, ou para pacientes com sintomas mínimos. Alguns autores têm defendido que o tumor desmoide apresenta um crescimento autolimitado e que a simples observação da lesão é suficiente, contraindicando o tratamento cirúrgico na maioria dos $\operatorname{casos}^{18}$. Esses pacientes podem ser seguidos clinicamente e com exames de imagem (preferencialmente tomografias computadorizadas) no intuito de se identificar um possível crescimento do tumor e uma possível compressão ureteral por tumores intra-abdominais.

A ressecção local, advogada desde muito tempo para o tratamento do tumor desmoide, tem sido o tratamento de escolha para a maioria dos autores. Essa ressecção deve ser ampla, atentando-se para as margens, já que normalmente esses tumores apresentam extensão não palpável para órgãos adjacentes, prejudicando a estimativa intraoperatória da extensão da doença ${ }^{19}$. Apesar de alguns autores terem relatado que o comprometimento da margem cirúrgica não altera a taxa de recidiva local ${ }^{20,21}$, outros têm mostrado claramente que o estado da margem cirúrgica é isoladamente o fator mais significante na determinação da taxa 
de recorrência local em pacientes tratados com cirurgia (recorrência local em torno de $54 \%$ nos casos de margem comprometida, contra $27 \%$ nos casos de margens livres) ${ }^{19}$. A taxa de recorrência local por tumor intra-abdominal é maior que a reportada por tumores extra-abdominais ou da parede abdominal, em torno de 57 a $86 \%{ }^{22}$, o que reflete um padrão de crescimento difuso e infiltrativo do tumor desmoide.

A ressecção de tumores superficiais da parede abdominal costuma ser fácil e segura ${ }^{9,15}$. A cirurgia em tumores intra-abdominais costuma ser mais agressiva e associada a aumento da morbidade e mortalidade. Assim, nesses casos, o tratamento cirúrgico apenas deve ser realizado quando os exames de imagem revelarem tumor que não acometa órgãos ou vasos vitais, em casos de resistência ao tratamento clínico ou em casos nos quais a cirurgia é a única possibilidade de tratamento. Caso contrário, biópsia incisional e terapia conservadora é preferível ${ }^{3}$.

Em geral, anti-inflamatórios não-hormonais e moduladores dos receptores de estrogênio (usualmente o tamoxifeno) são considerados primeira linha no tratamento farmacológico ${ }^{22,23}$. O tumor desmoide não necessita ter receptor de estrogênio positivo para responder ao tamoxifeno ${ }^{10}$. Inibidores de aromatase também parecem ter efeito importante na doença, mas futuros estudos se fazem necessários para se atestar tal questão, já que, atualmente, não há estudos randomizados que comprovem sua eficácia. Aproximadamente metade dos tumores desmoides apresenta resposta completa ou parcial à terapia farmacológica, porém, é difícil comprovar se isso se deve verdadeiramente ao tratamento. A terapia farmacológica pode ser usada em conjunto com o tratamento cirúrgico, como um tratamento adjuvante, mas não existem indícios científicos de que tal modalidade de terapia reduza a recorrência tumoral, principalmente naqueles pacientes com doença intra-abdominal avançada ${ }^{9}$.

$\mathrm{O}$ uso de quimioterapia também tem se mostrado controverso ${ }^{24}$. Recentemente validada ${ }^{25}$, tal modalidade de tratamento pode ser considerada com indicações específicas, normalmente após falência no tratamento não-citotóxico em pacientes com tumores não operáveis ou irressecáveis (por exemplo, tumores extensos retroperitoneais envolvendo estruturas vitais como grandes vasos, nervos e ureteres) ou em pacientes com doença residual ${ }^{9,13,15}$. Além disso, a quimioterapia pode ser usada na forma neoadjuvante, no intuito de diminuir o tamanho do tumor no pré-operatório, ou de forma paliativa. O regime quimioterápico mais efetivo e de menor toxicidade é a combinação de metotrexate e vimblastina ou doxorrubicina e dacarbazina. As taxas de resposta completa, em regimes baseados em doxorrubicina, variam em torno de $50 \%$, porém sua toxicidade deve merecer uma especial atenção ${ }^{15}$.

O uso da radioterapia no tratamento do tumor desmoide é também controverso. Não se sabe se há benefícios significantes no uso da radioterapia pós-tratamento cirúrgico de lesões com margem comprometidas, no intuito de se evitarem recidivas ${ }^{21,24}$. No entanto, a radioterapia tem sido usada em pacientes com doença irressecável ou, eventualmente, como terapia adjuvante após ressecções incompletas do tumor ${ }^{11,19}$.

Assim como é escolha da maioria dos autores, a paciente por nós relatada foi tratada exclusivamente com cirurgia, ressecando-se completamente a lesão, sem evidência de margem comprometida. Após acompanhamento clínicoradiológico por um período de seis anos, a paciente não apresenta qualquer sinal de recidiva da lesão.

\section{Referências}

1. Schlemmer M. Desmoid tumors and deep fibromatoses. Hematol Oncol Clin North Am. 2005;19(3):565-71, vii-viii.

2. Mendenhall WM, Zlotecki RA, Morris CG, Hochwald SN, Scarborough MT. Aggressive fibromatosis. Am J Clin Oncol. 2005;28(2):2 11 1-5.

3. Rampone B, Pedrazzani C, Marrelli D, Pinto E, Roviello F. Updates on abdominal desmoid tumors. World J Gastroenterol. 2007; 13(45):5985-8.

4. Wong SL. Diagnosis and management of desmoid tumors and fibrosarcoma. J Surg Oncol. 2008;97(6):554-8.

5. Cholongitas E, Koulenti D, Panetsos G, Kafiri G, Tzirakis E, Thalasinou $P$, et al. Desmoid tumor presenting as intra-abdominal abscess. Dig Dis Sci. 2006;51(1):68-9.

6. Murayama T, Imoto S, Ito M, Matsushita K, Matozaki S, Nakagawa $\mathrm{T}$, et al. Mesenteric fibromatosis presenting as fever of unknown origin. Am J Gastroenterol. 1992;87(10):1503-5.
7. Owens CL, Sharma R, Ali SZ. Deep fibromatosis (desmoid tumor): cytopathologic characteristics, clinicoradiologic features, and immunohistochemical findings on fine-needle aspiration. Cancer. 2007; 111 (3):166-72.

8. Papagelopoulos PJ, Mavrogenis AF, Misiokapa EA, Papaparaskeva KT, Galanis EC, Soucacos PN. Current trends in the management of extra-abdominal desmoid tumours. World J Surg Oncol. 2006:4:21-8.

9. Latchford AR, Sturt NJ, Neale K, Rogers PA, Phillips RK. A 10-year review of surgery for desmoid disease associated with familial adenomatous polyposis. Br J Surg. 2006;93(10):1258-64.

10. Sakorafas $G H$, Nissotakis $C$, Peros $G$. Abdominal desmoid tumors. Surg Oncol. 2007;16(2):131-42.

11. Reitamo JJ, Scheinin TM, Hayry P. The desmoid syndrome: new aspects in the cause, pathogenesis and treatment of the desmoid tumor. Am J Surg. 1986;151 (2):230-7. 
12. Rodriguez-Bigas MA, Mahoney MC, Karakousis CP, Petrelli NJ. Desmoid tumors in patients with familial adenomatous polyposis. Cancer. 1994;74(4):1270-4.

13. Church J, Berk T, Boman BM, Guillem J, Lynch C, Lynch P, et al. Staging intra-abdominal desmoid tumors in familial adenomatous polyposis: a search for a uniform approach to a troubling desease. Dis Colon Rectum. 2005;48(8):1528-34.

14. Lee JC, Thomas JM, Phillips S, Fisher C, Moskovic E. Aggressive fibromatosis: MRI features with pathologic correlation. AJR Am J Roentgenol. 2006; 186(1):247-54.

15. Sturt NJ, Clark SK. Current ideas in desmoid tumours. Fam Cancer. 2006;5(3):275-85.

16. Hartley JE, Church JM, Gupta S, McGannon E, Fazio VW. Significance of incidental desmoids identified during surgery for familial adenomatous polyposis. Dis Colon Rectum. 2004;47(3):334-8.

17. Overhaus M, Decker P, Fischer HP, Textor HJ, Hirner A. Desmoid tumors of the abdominal wall: a case report. World J Surg Oncol. $2003 ; 1(1): 11$.

18. Nakayama T, Tsuboyama T, Toguchida J, Hosaka T, Nakamura T. Natural course of desmoid-type fibromatosis. J Orthop Sci. 2008;13(1):51-5.

19. Ballo MT, Zagars GK, Pollack A, Pisters PW, Pollack RA. Desmoid tumor: prognostic factors and outcome after surgery, radiation therapy, or combined surgery and radiation therapy. J Clin Oncol. $1999 ; 17(1): 158-67$

20. Merchant BN, Lewis JJ, Woodruff JM, Leung DH, Brennan MF. Extremity and trunk desmoid tumors: a multifactorial analysis of outcome. Cancer. 1999;86(10):2045-52.

21. Gronchi A, Casali PG, Mariani L, Lo Vullo S, Colecchia M, Lozza $L$, et al. Quality of surgery and outcome in extra-abdominal aggressive fibromatosis: a series of patients surgically treated at a single institution. J Clin Oncol. 2003;21(7):1390-7.

22. Tamura K, Tani M, Kinoshita H, Yamaue H. Mesenteric desmoid tumor of the interposed jejunal pouch after total gastrectomy. World J Surg Oncol. 2006;4(1):27-31.

23. Tanaka K, Yoshikawa R, Yanagi H, Gega M, Fujiwara Y, HashimotoTamaoki $T$, et al. Regression of sporadic intra-abdominal desmoid tumour following administration of non-steroidal anti-inflammatory drug. 2008;6(1):17.

24. Lev D, Kotilingam D, Wei C, Ballo MT, Zagars GK, Pisters PW, et al. Optimizing treatment of desmoid tumors. J Clin Oncol. 2007;25(13):1785-91.

25. Gega M, Yanagi H, Yoshikawa R, Noda M, Ikeuchi H, Tsukamoto $K$, et al. Successful chemotherapeutic modality of doxorubicin plus dacarbazine for the treatment of desmoid tumors in association with familial adenomatous polyposis. J Clin Oncol. 2006;24(1):102-5. 